\title{
Identificação e quantificação de indicadores-referência de sistemas de produção de leite
}

\section{André Soares de Oliveira ${ }^{1}$, Daniel de Noronha Figueiredo Vieira da Cunha ${ }^{1}$, José Maurício de Souza Campos², Sônia Maria Leite Ribeiro do Vale ${ }^{3}$, Anderson Jorge de Assis ${ }^{4}$}

\author{
${ }^{1}$ Doutorando em Nutrição e Produção de Ruminantes, Departamento de Zootecnia/UFV. Bolsista do CNPq. \\ 2 Departamento de Zootecnia/UFV, Viçosa-MG. \\ 3 Departamento de Economia Rural/UFV, Viçosa-MG. \\ ${ }^{4}$ D.Sc. em Nutrição e Produção de Ruminantes/UFV.
}

RESUMO - Objetivou-se identificar e quantificar indicadores-referência de sistemas de produção de leite no Extremo Sul da Bahia. Analisaram-se o perfil tecnológico e os indicadores zootécnicos, econômicos e de tamanho de nove empresas. Foram determinados os coeficientes de correlação dos indicadores com a taxa de remuneração do capital investido. Após a identificação dos indicadores que apresentaram correlação, foram geradas equações de regressão para cada indicador em função da taxa de remuneração do capital investido para quantificar os indicadores-referência em quatro cenários de taxa de remuneração do capital investido (4, 6, 8 e $10 \%$ ao ano). Os indicadores correlacionados e seus respectivos valores nos quatro cenários foram: produção diária de leite $(456,538,621$ e $703 \mathrm{~L} /$ dia); produtividade da terra $(733,1.008,1.284$ e $1.559 \mathrm{~L} / \mathrm{ha} / \mathrm{ano})$; vacas em lactação por área $(0,37 ; 0,45 ; 0,54$ e 0,62 vacas/ha); produtividade por total de vacas $(3,01 ; 3,52 ; 4,03$ e $4,54 \mathrm{~L} / \mathrm{vaca} / \mathrm{dia})$; relação de vacas em lactação pelo total do rebanho (24, 27, 30 e 33\%); produtividade da mão-de-obra $(111,124,137$ e $150 \mathrm{~L} /$ dia-homem), participação do custo operacional efetivo da atividade na renda bruta da atividade (60, 57, 54 e 51\%); participação do custo operacional total da atividade na renda bruta da atividade (72, 66, 60, 54\%); gasto com mão-de-obra em relação à renda bruta do leite (31,27, 22 e 18\%); capital investido na atividade em relação à produção diária de leite (2.093, 1.508, 924 e 339 R \$/L-dia). A identificação de indicadores-referência em sistemas reais de produção de leite, caracterizando aqueles mais diretamente correlacionados à eficiência econômica, é uma importante ferramenta de apoio gerencial e pode trazer esclarecimentos para o debate sobre a viabilidade econômica de sistemas de produção de leite.

Palavras-chave: benchmark, custo de produção, gestão, rentabilidade

\section{Identification and quantification of benchmarks of milk production systems}

\begin{abstract}
The objective of this study was to identify and quantify benchmarks of milk production systems in the region of Extremo Sul da Bahia. Technological profile and indicators of size, livestock productivity and economy of nine farms were analyzed. It were also determined the correlation coefficients between these indicators and the rate of retun. Indicators with significant correlation were regressed on rate of return in order to quantify the benchmarks with the following rates of return: 4, 6, 8 and $10 \%$ per year. Data for the four rates of return were, respectively: daily milk yield (456, 538, 621 and $703 \mathrm{~L} /$ day); land productivity $(733,1.008,1284$ and $1559 \mathrm{~L} / \mathrm{ha} /$ year $)$; stocking rate $(0.37,0.45,0.54$ and $0.62 \mathrm{cows} / \mathrm{ha})$; productivity per total of cows $(3.01,3.52,4.03$ and $4.54 \mathrm{~L} / \mathrm{cow} /$ day $)$; labor productivity $(111,124,137 \mathrm{and} 150 \mathrm{~L} / \mathrm{man} / \mathrm{day})$; proportion of the total effective operational cost in the gross revenue $(60,57,54$ and $51 \%)$; proportion of the total operational cost in the gross revenue $(72,66,60$ and $54 \%)$; labor cost to milk gross revenue ratio $(31,27,22$ and $18 \%)$; and capital invested to daily milk production ratio $(2093,1508,924$ and $339 \mathrm{R} \$ / \mathrm{L}$-day). Identification of benchmarks, mainly those highly correlated with economic efficiency is an important tool for applying on farm management because help producers to better understand the economic viability of their dairy production systems.
\end{abstract}

Key Words: benchmark, production cost, administration, profitability

\section{Introdução}

No ambiente de elevada concorrência, de incertezas e de redução das margens de ganho em que os produtores de leite estão inseridos, a eficácia nas decisões é fundamental para competitividade do negócio.
O planejamento é essencial para o gerenciamento de decisões operacionais, táticas e estratégicas. Entre os métodos de planejamento, a identificação e a análise de pontos de referência (benchmark) destacam-se pela segurança e exatidão, visto que os valores são obtidos diretamente de unidades de produção presentes em mesmo 
ambiente econômico (Gomes, 2005b). Neste sentido, estudos têm sido realizados visando identificar os principais indicadores zootécnicos e econômicos que influenciam a rentabilidade dos sistemas de produção de leite no Brasil (Krug, 2001; Gomes, 2005b).

Entretanto, o caráter dinâmico inerente ao ambiente de produção e a elevada diversidade socioeconômica, cultural e edafo-climática que caracterizam os sistemas de produção, associados ao fato de a pecuária leiteira estar presente em mais de $80 \%$ dos municípios do Brasil, impõem a necessidade de estudos regionalizados.

Com este trabalho objetivou-se identificar e quantificar indicadores-referência de sistemas de produção de leite no Extremo SuldaBahia, maior bacia leiteiradoestado(IBGE, 2005).

\section{Material e Métodos}

Para identificar e quantificar os indicadores-referência, avaliaram-se nove empresas produtoras de leite, localizadas no Extremo Sul da Bahia (Microrregião de Porto Seguro, segundo o IBGE - Instituto Brasileiro de Geografia e Estatística), participantes de um programa de assistência técnica e gerencial. Os dados originaram-se de registros mensais, realizados entre janeiro e dezembro de 2002, cujos valores monetários foram corrigidos para janeiro de 2005 pelo IGP-DI (índice geral de preços de disponibilidade interna) (FGVDADOS, 2005).

Segundo classificação de Köppen, o clima da região é o tropical úmido (Athi), caracterizado como megatérmico, sempre úmido, sem estação seca, com temperaturas médias mensais sempre superiores a $18^{\circ} \mathrm{C}$, amplitude térmica menor que $5^{\circ} \mathrm{C}$ e precipitação média anual de $1.400 \mathrm{~mm}$ (Hídricos, 2003).

Amostras mensais de leite foram coletadas de cada propriedade, diretamente do tanque de expansão, para contagem padrão em placas e contagem de células somáticas. As análises foram realizadas pelo laboratório da agroindústria receptora do leite.

As avaliações fundamentaram-se na teoria neoclássica de produção, em que as firmas buscam maximizacão dos lucros ou minimização dos custos mediante combinações ótimas de fatores de produção e de insumos, sujeitos a determinados vetores tecnológicos (Ferguson, 1996). Os vetores tecnológicos, por sua vez, foram predeterminados a partir da seleção de indicadores-referência (benchmarks).

Foram analisados o perfil tecnológico, os indicadores zootécnicos, econômicos e de tamanho das empresas utilizando-se planilhas do Programa Excel ${ }^{\circledR}$.

Os indicadores de tamanho analisados foram: produção diária de leite (litros); área total (ha); número de vacas em lactação (animais), total de vacas (animais), total do rebanho (animais) e total de unidades animais (UA), mãode-obra total (dias-homem/ano), fornecimento de concentrado para o rebanho ( $\mathrm{kg} / \mathrm{ano}$ ) e capital total investido (R\$) (terra, animais, benfeitorias, máquinas e forrageiras não-anuais).

Os indicadores zootécnicos analisados foram: 1 - produtividade por vaca em lactação $($ litros/vaca/dia $)=$ produção diária de leite/número de vacas em lactação; 2 - produtividade por total de vacas (litros/vaca/dia) = produção diária de leite/número total de vacas; 3 - relação de vacas em lactação pelo total de vacas $(\%)=$ número de vacas em lactação/número total de vacas; 4 - relação de vacas em lactação pelo total do rebanho $(\%)=$ número de vacas em lactação/total do rebanho; 5 - taxa de lotação $(\mathrm{UA} / \mathrm{ha})=\mathrm{n} \underline{\mathrm{o}}$ total de unidade animais/área total; 6 - número de vacas em lactação por área $($ vacas $/ \mathrm{ha})=\mathrm{n} \underline{\mathrm{o}}$ de vacas em lactação $/$ área total; 7 - produtividade da terra $($ litros/ha/ano $)=$ produção anual de leite / área total; 8 - produtividade da mão-de-obra (litros/dia-homem $)=$ produção anual de leite/total de mãode-obra anual; 9 - produtividade do concentrado (litros / kg de matéria natural $)$ = produção anual de leite/fornecimento anual de concentrado para o rebanho. A medodologia de cálculo de custo se baseou nos métodos de custo operacional (Hoffman et al., 1987) e de custo total. Os indicadores analisados foram: 10 - renda bruta da atividade leiteira-RBA $(\mathrm{R} \$ / \mathrm{ano})=$ venda de leite + venda de animais; 11 - renda bruta do leite - RBL (R\$/ano) = venda de leite; 12 - participação da renda bruta do leite na renda bruta da atividade $(\%)=\mathrm{RBL} / \mathrm{RBA} \times 100 ; 13-$ preço do leite $(\mathrm{R} \$ /$ litro $)=$ valor unitário do leite recebido, incluíndo frete; 14 - custo operacional efetivo da atividade leiteira - COE $(\mathrm{R} \$ / \mathrm{ano})=$ mão-de-obra contratada, concentrados, manutenção de forrageiras não-anuais, mineralização, sanidade, energia e combustíveis, material de ordenha, inseminação artificial, frete de leite, impostos e taxas, reparos em benfeitorias e máquinas e outras despesas de custeio; 15 custo operacional total da atividade leiteira-COT ( $\$$ \$ano) $=\mathrm{COE}+$ mão-de-obra familiar + depreciação de máquinas, benfeitorias, forrageiras não-anuais e animais de serviços - para o cálculo da depreciação, foi utilizado o método linear (Hoffman et al., 1987); 16 - custo total da atividade leiteira$\mathrm{CT}(\mathrm{R} \$ / \mathrm{ano})=\mathrm{COT}+$ remuneração do capital médio investido em animais, benfeitorias, máquinas, forrageiras nãoanuais e terras - para o cálculo da remuneração do capital investido, adotou-se taxa de juros de $4 \%$ ao ano, referente à remuneração média real (nominal descontada a inflação) da caderneta de poupança entre os anos de 1995 a 2004; 17 - margem bruta da atividade $-\mathrm{MB}(\mathrm{R} \$ / \mathrm{ano})=\mathrm{RBA}-\mathrm{COE}$; 18 - margem líquida da atividade-ML $(\mathrm{R} \$ / \mathrm{ano})=\mathrm{RBA}-$ 
COT; 19 - lucro da atividade $(\mathrm{R} \$ / \mathrm{ano})=\mathrm{RBA}-\mathrm{CT} ; 20$ custo operacional efetivo por litro de leite $(\mathrm{R} \$ /$ litro $)=$ (COE X (RBL / RBA x 100)) / produção anual de leite; 21 custo operacional total por litro de leite $(\mathrm{R} \$ /$ litro $)=(\mathrm{COT}$ $\mathrm{X}(\mathrm{RBL} / \mathrm{RBA} \times \mathrm{100})$ ) / produção anual de leite; 22 - custo total por litro de leite $(\mathrm{R} \$ /$ litro $)=(\mathrm{CT} \mathrm{X}(\mathrm{RBL} / \mathrm{RBA} \times 100))$ / produção anual de leite; 23 - margem bruta por litro de leite $(\mathrm{R} \$ /$ litro $)=$ preço do leite $-\mathrm{COE}$ do leite; 24 - margem líquida por litro de leite $(\mathrm{R} \$ /$ litro $)=$ preço do leite $-\mathrm{COT}$ do leite; 25 - lucro por litro de leite $(\mathrm{R} \$ /$ litro $)$ = preço do leite - CT do leite; 26 - gasto com concentrado para o rebanho em relação ao valor da produção de leite $(\%)=$ gasto anual com concentrado para o rebanho/RBL x 100;27 - gasto com mão-de-obra em relação ao valor da produção de leite (\%) = gasto anual com mão-de-obra/RBL x 100; 28 - participação do COE na RBA $(\%)=$ COE / RBA x 100;29 - participação do COT na RBA $(\%)=\mathrm{COT} / \mathrm{RBA} \times 100 ; 30$ - taxa de remuneração do capital investido (\% ao ano $)=\mathrm{ML} /$ capital médio investido em animais, benfeitorias, máquinas, forrageiras não-anuais e terra; 31 - capital investido na atividade em relação à produção diária de leite $(\mathrm{R} \$ /$ litro/dia) = capital médio investido em animais, benfeitorias, máquinas, forrageiras não-anuais e terra / produção diária de leite. Foram determinados os coeficientes de correlação dos indicadores de tamanho, zootécnicos e econômicos com a taxa de remuneração do capital investido, com o objetivo de identificar aqueles que afetam o desempenho econômico das empresas. Adotou-se o procedimento de correlação Pearson, aplicando-se o teste $\mathrm{t}$ a $10 \%$ de probabilidade.

Após a identificação dos indicadores que apresentaram correlação $(\mathrm{P}<0,10)$, foram geradas equações de regressão para cada indicador em função da taxa de remuneração do capital investido, para quantificar os indicadoresrefe rência. Os valores de cada indicador foram estimados considerando quatro cenários de taxa real de remuneração do capital investido (4, 6, 8 e 10\% ao ano).

Os procedimentos estatísticos adotados (análise de variância e regressão) foram realizados utilizando-se o programa SAEG, versão 7.0. (UFV, 1997).

\section{Resultados e Discussão}

Os sistemas de produção analisados caracterizaram-se pela utilização de rebanhos com composição genética entre 1/4 e 3/4 Holandês x Zebu (HZ), com predominância dos grupos genéticos $1 / 2$ e $5 / 8(\mathrm{HZ})$, e pelo aproveitamento dos machos, comercializados com dez meses de idade.

Na Tabela 1 são apresentados os perfis tecnológico e gerencial das empresas que exploram a pecuária leiteira no Extremo Sul da Bahia.
Apesar de a maioria (81\%) adotar a prática de aleitamento natural, o que poderia caracterizar sistemas de baixo nível tecnológico, todos os produtores utilizaram inseminação artificial e $92 \%$ duas ordenhas diárias. Esse resultado é reflexo da composição genética do rebanho, que exige a presença do bezerro durante a ordenha, a fim de evitar redução no período de lactação (Oliveira et al., 2004).

Como estratégia de alimentação volumosa, no período da ságuas, a pastagem foi utilizada como volumoso exclusivo. O pastejo rotativo foi utilizado por $41 \%$ dos produtores, frequiência considerada alta se comparada à de outras regiões do país (Bressan et al., 1999). No período seco do ano, todos os produtores utilizaram cana-de-açúcar como volumoso suplementar.

Os sistemas de controles leiteiro, reprodutivo e financeiro foram adotados por todos os produtores, evidenciando a importância atribuída aos sistemas de informação como recurso de gestão.

Apesar da baixa adoção de ordenha mecânica (16\%), o resfriamento do leite em tanques de expansão direta foi utilizado em todas as unidades produtivas, o que possivelmente contribuiu para obtenção de contagem padrão em placa (CPP) de 305 mil unidades formadoras de colônia (ufc)

Tabela 1 - Perfil tecnológico e gerencial das empresas que exploram pecuária leiteira no Extremo Sul da Bahia

Table 1- Technological and managerial profile of dairy farms in the Extremo Sul da Bahia

\begin{tabular}{|c|c|}
\hline $\begin{array}{l}\text { Especificação } \\
\text { Item }\end{array}$ & $\begin{array}{l}\text { ia de utilização (\%) } \\
\text { Frequency }\end{array}$ \\
\hline Participação da mão-de-obra familiar & 8 \\
\hline $\begin{array}{l}\text { Proportion of family labor } \\
\text { Duas ordenhas diárias } \\
\text { Two daily milkings }\end{array}$ & 92 \\
\hline Aleitamento natural & 81 \\
\hline $\begin{array}{l}\text { Natural nursing } \\
\text { Inseminação artificial }\end{array}$ & 100 \\
\hline $\begin{array}{l}\text { Artificial insemination } \\
\text { Fornecimento de concentrado }\end{array}$ & 47 \\
\hline Concentrate feeding & \\
\hline $\begin{array}{l}\text { Fornecimento de volumoso } \\
\text { suplementar - cana-de-açúcar }\end{array}$ & 100 \\
\hline Feeding of supplementary forage - sugarcane & \\
\hline Pastejo rotativo & 41 \\
\hline $\begin{array}{l}\text { Rotational grazing } \\
\text { Adubação de pastagens }\end{array}$ & 61 \\
\hline $\begin{array}{l}\text { Pasture fertilization } \\
\text { Controle leiteiro }\end{array}$ & 100 \\
\hline Milk production control & \\
\hline Controle reprodutivo & 100 \\
\hline $\begin{array}{l}\text { Reproductive control } \\
\text { Controle financeiro }\end{array}$ & 100 \\
\hline $\begin{array}{l}\text { Financial control } \\
\text { Resfriamento do leite por expansão direta } \\
\text { Milk cooling tank }\end{array}$ & 100 \\
\hline $\begin{array}{l}\text { Ordenha mecânica } \\
\text { Mechanical milking }\end{array}$ & 16 \\
\hline
\end{tabular}


por $\mathrm{mL}$, indicando que não houve relação direta entre utilização de ordenha mecânica e a qualidade do leite, visto que os valores de CPP atenderam aos limites máximos de 750 mil ufc/mL exigidos pela Instrução Normativa no 51 do MAPA até 2012 (Dürr, 2005). O valor médio de contagem de células somáticas (CCS) foi de 223.000/mL, o que atende às normas nacionais e internacionais, que limitam os valores em 400.000 por mL (Ribas et al., 2003; Dürr, 2005).

As estatísticas descritivas dos indicadores de tamanho de empresas que exploram pecuária leiteira no Extremo Sul da Bahia encontram-se relacionadas na Tabela 2. A produção diária de leite - média de 521,08 e mínimo de $273,51 \mathrm{~L}$ - inclui os produtores no grupo acima de $200 \mathrm{~L} /$ dia, que, segundo o Censo das Cooperativas de Laticínios do Brasil em 2002, apesar de responder por $23 \%$ do número total de produtores, contribui com $68 \%$ da produção (Alvarez et al., 2003).

A área total utilizada pela pecuária leiteira indica que o tamanho das propriedades pode ser considerado elevado em comparação ao das propriedades que exploram a atividade nos estados de Minas Gerais, 92,85 ha (Diagnóstico da Pecuária Leiteira do Estado de Minas Gerais, 1996), e Goiás, 124,4 ha (Bressan et al., 1999). Além disso, 80\% da produção de leite no Brasil é oriunda de propriedades com menos de 200 ha (IBGE, 1996).

$\mathrm{Na}$ Tabela 3 constam as estatísticas descritivas dos indicadores zootécnicos de empresas que exploram pecuária leiteira no Extremo Sul da Bahia. A produtividade por vaca em lactação observada (2.146,2 L/vaca/ano) é compatível com o sistema de produção adotado, caracterizado pela baixa utilização de concentrados e pela opção de sistema de cruzamento alternado simples (HZ) (Madalena et al., 1990).

A relação de vacas em lactação pelo total de vacas é um indicador influenciado pela razão entre o período de lactação e o intervalo de partos. Considerando que o período médio de lactação foi de 280 dias, o intervalo de partos do grupo foi de 487 dias.

A relação de vacas em lactação pelo total do rebanho é um índice global do sistema de produção, pois, além de ser afetado pelo intervalo de partos e pelo período de lactação, também sofre influência negativa da idade ao primeiro parto. Maiores valores indicam maiores proporções de animais gerando receitas em relação ao número total do rebanho.

O número de vacas em lactação por área e a produtividade da terra são indicadores de intensificação do sistema de produção relacionados às tecnologias bioquímicas poupadoras do fator terra, mais relevantes em regiões onde o preço desse fator é elevado. Os valores obtidos caracterizam os sistemas como extensivos no uso deste recurso produtivo (Gomes, 2005c).

A produtividade da mão-de-obra é considerada também fator de intensificação, mas reflexo do uso de tecnologias mecânicas, poupadoras de mão-de-obra (Gomes, 2005c). Em condições crescentes de custos com mão-de-obra, a intensificação desse fator é necessária para o equilíbrio econômico do sistema de produção.

A produtividade do concentrado pode ser considerada elevada se comparada aos dados médios de $2,73 \mathrm{~L}$ de leite por $\mathrm{kg}$ de concentrado observados no estado de Minas Gerais DIAGNÓSTICO DA PECUÁRIA LEITEIRA DO

Tabela 2 - Estatísticas descritivas dos indicadores de tamanho de empresas que exploram pecuária leiteira no Extremo Sul da Bahia Table 2 - Descriptive statistics of indicators of size of dairy farms in the Extremo Sul da Bahia

\begin{tabular}{|c|c|c|c|c|c|}
\hline $\begin{array}{l}\text { Especificação } \\
\text { Item }\end{array}$ & $\begin{array}{l}\text { Unidade } \\
\text { Unity }\end{array}$ & $\begin{array}{l}\text { Média } \\
\text { Mean }\end{array}$ & $\begin{array}{l}\text { Mínimo } \\
\text { Minimum }\end{array}$ & $\begin{array}{l}\text { Máximo } \\
\text { Maximum }\end{array}$ & $\begin{array}{l}\mathrm{DP} \\
S D\end{array}$ \\
\hline 1 & L/ano (L/year) & $190.194,20$ & $99.831,15$ & $263.895,00$ & $51.194,90$ \\
\hline 2 & $\mathrm{~L} / \mathrm{dia}$ (L/year) & 521,08 & 273,51 & 723,00 & 140,26 \\
\hline 3 & ha & 212,44 & 130,00 & 330,00 & 62,49 \\
\hline 4 & $\mathrm{Cab}$ (cows) & 88,56 & 47,00 & 120,00 & 27,21 \\
\hline 5 & $\mathrm{Cab}$ (cows) & 154,11 & 105,00 & 212,00 & 34,04 \\
\hline 6 & Cab (head) & 331,67 & 211,00 & 420,00 & 71,06 \\
\hline 7 & U.A. & 240,33 & 152,00 & 307,00 & 53,23 \\
\hline 8 & d.h./ano & $1.612,89$ & $2.054,00$ & $2.547,00$ & 504,40 \\
\hline 9 & $\mathrm{~kg} / \mathrm{ano}$ (kg/year) & $15.612,50$ & $1.800,00$ & $31.700,00$ & $11.948,64$ \\
\hline 10 & $\mathrm{R} \$$ & $789.594,00$ & $543.247,00$ & $1.056 .056,00$ & $157.908,48$ \\
\hline
\end{tabular}

1 - Produção anual de leite (Annual milk production).

2 - Produção diária de leite(Daily milk production).

3 - Área total (Total area).

4 - Número de vacas em lactação (Number of lactating cows).

5 - Número total de vacas (Total number of cows).

6 - Total do rebanho (Total herd).

7 - Total de unidades animais (Total amounts of animals).

8 - Mão-de-obra total (Total labor).

9 - Fornecimento anual de concentrados (Annual supply of concentrate).

10 - Capital investido com terra (Capital invested on land). 
Tabela 3 - Estatísticas descritivas dos indicadores zootécnicos de empresas que exploram pecuária leiteira no Extremo Sul da Bahia Table 3 - Descriptive statistics of herd indicators of dairy farms in the Extremo Sul da Bahia

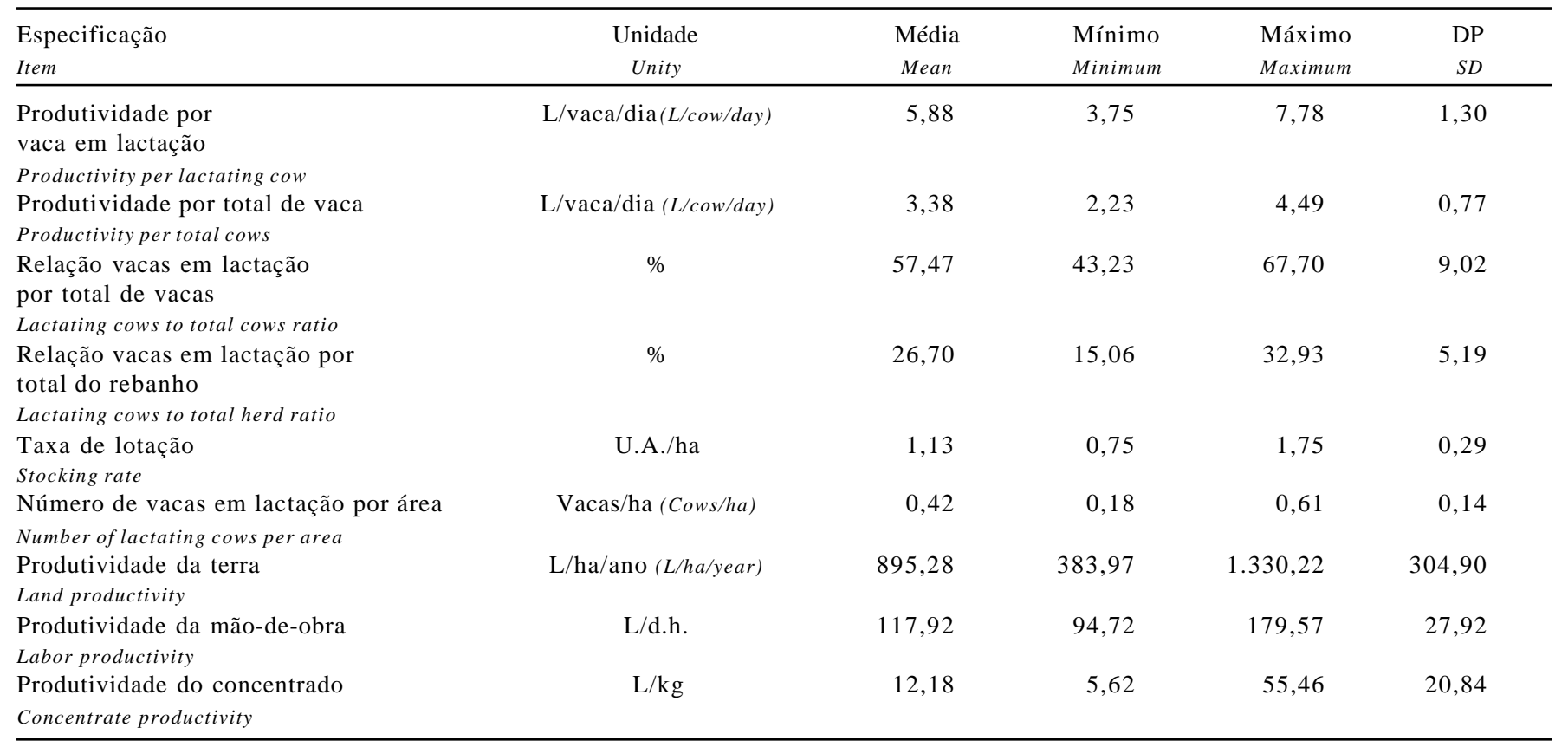

ESTADO DE MINAS GERAIS, 1996). Este resultado se deve ao menor nível de utilização, visto que a resposta produtiva do concentrado ( $\mathrm{kg}$ de leite/kg de concentrado) decresce com o aumento da quantidade fornecida (Davison \& Elliott, 1993; Lana et al., 2005). Por sua vez, o baixo nível de utilização de concentrado deveu-se à relação de troca desfavorável de preços $(1,02 \mathrm{~kg}$ de concentrado por litro de leite), situando-se acima dos valores de respostas produtivas ao uso de concentrados, entre 0,5 e $1,0 \mathrm{~kg}$ de leite por kg de concentrado (Davison \& Elliott, 1993; Gomide, 1993; Deresz, 2001; Bargo et al., 2003; Lana, 2005).

Nas Tabelas 4 e 5 são apresentadas as estatísticas descritivas dos custos de produção, da renda bruta, da margem bruta, da margem líquida e dos lucros de empresas que exploram pecuária leiteira na região do Extremo Sul da Bahia. A participação da renda bruta do leite na composição da renda bruta da atividade leiteira indica a importância da venda de animais, característica desse sistema de pro dução.

A participação do gasto com concentrado e mão-deobra na renda bruta do leite consiste em um indicador de eficiência econômica largamente adotado. Observou-se maior participação da mão-de-obra na renda bruta do leite em relação à participação do concentrado, o que difere de sistemas nos quais se utilizam vacas de maior nível de produção de leite, em decorrência da maior adoção de tecnologias poupadoras de mão-de-obra e da maior intensidade de uso de concentrado neste tipo de sistema de produção.
O conhecimento das implicações do custo operacional efetivo (COE), a curto prazo, do custo operacional total (COT), a médio prazo, e do custo total (CT), a longo prazo, é fundamental na gestão do negócio. A associação temporal do COE indica a viabilidade financeira a curto prazo; e o COT e o CT representam a sustentabilidade do negócio a médio e longo prazos. Neste sentido, a empresa deve sempre manter a margem bruta positiva, pois, caso contrário, a interrupção da produção será a melhor alternativa.

Margem líquida nula (ponto de resíduo) implica recomposição do desgaste, pelo tempo ou pelo avanço tecnológico, de benfeitorias, máquinas e de forrageiras não-anuais e na remuneração da mão-de-obra familiar, mas não remunera o capital investido na atividade. Valores positivos indicam que o capital está sendo remunerado a taxas que podem ser menores, iguais ou maiores que a taxa de juros de oportunidade adotada, sendo esta interpretação dependente dos resultados de lucro.

Em situações de lucro negativo, a taxa de remuneração do capital investido será menor que a de juros de oportunidade. Em situações de lucro positivo (lucro supernormal), a taxa de remuneração do capital investido será maior que a taxa de juros de oportunidade. Quando o lucro é zero (lucro normal), o negócio remunera o capital a taxa equivalente aos juros de oportunidade, configurando uma situação de equilíbrio.

Considerando que os produtores de leite se situam em um ambiente próximo à concorrência perfeita, caracterizado pela ausência de influência individual no mercado e pela 
Tabela 4 - Estatísticas descritivas dos custos de produção e da renda bruta de empresas que exploram pecuária leiteira no Extremo Sul da Bahia

Table 4 - Descriptive statistics of the gross income and production costs of dairy farms in the Extremo Sul da Bahia

\begin{tabular}{|c|c|c|c|c|c|}
\hline $\begin{array}{l}\text { Especificação } \\
\text { Item }\end{array}$ & $\begin{array}{l}\text { Unidade } \\
\text { Unity }\end{array}$ & $\begin{array}{l}\text { Média } \\
\text { Mean }\end{array}$ & $\begin{array}{l}\text { Mínimo } \\
\text { Minimum }\end{array}$ & $\begin{array}{l}\text { Máximo } \\
\text { Maximum }\end{array}$ & $\begin{array}{l}\mathrm{DP} \\
S D\end{array}$ \\
\hline 1 & $\mathrm{R} \$ /$ ano $(R \$ /$ year $)$ & $125.603,22$ & $70.538,00$ & $165.636,00$ & $33.486,33$ \\
\hline 2 & $\mathrm{R} \$$ /ano ( $R \$ /$ year) & $92.572,67$ & $48.554,00$ & $128.402,00$ & $24.939,66$ \\
\hline 3 & $\%$ & 73,70 & 65,36 & 83,92 & 6,31 \\
\hline 4 & $\mathrm{R} \$ / \mathrm{L}$ & 0,4867 & 0,4867 & 0,4867 & 0,00 \\
\hline 5 & $\mathrm{R} \$$ /ano ( $R$ \$/year) & $7.838,15$ & $1.213,85$ & $18.403,35$ & $6.327,38$ \\
\hline 6 & $\mathrm{R} \$$ /ano ( $R \$ /$ year) & $25.144,05$ & $17.158,23$ & $35.952,56$ & $6.908,53$ \\
\hline 7 & $\%$ & 8,47 & 1,50 & 17,0 & 6,11 \\
\hline 8 & $\%$ & 27,16 & 20,00 & 41,00 & 5,95 \\
\hline 9 & $\mathrm{R} \$$ /ano ( $R \$ /$ year) & $71.798,67$ & $46.175,00$ & $102.085,00$ & $20.361,25$ \\
\hline 10 & $\mathrm{R} \$$ /ano ( $R \$ /$ year) & $83.175,22$ & $59.025,00$ & $112.092,00$ & $21.305,88$ \\
\hline 11 & $\mathrm{R} \$$ /ano ( $R \$ /$ year) & $114.759,00$ & $85.036,00$ & $147.326,00$ & $24.509,43$ \\
\hline 12 & $\%$ & 57,16 & 45,00 & 65,00 & 5,74 \\
\hline 13 & $\%$ & 66,22 & 56,00 & 84,00 & 7,52 \\
\hline 14 & $\mathrm{R} \$ / \mathrm{L}$ & 0,2782 & 0,2207 & 0,3184 & 0,0276 \\
\hline 15 & $\mathrm{R} \$ / \mathrm{L}$ & 0,3223 & 0,2742 & 0,4070 & 0,0357 \\
\hline 16 & $\mathrm{R} \$ / \mathrm{L}$ & 0,4447 & 0,3885 & 0,6482 & 0,0791 \\
\hline
\end{tabular}

1 - Renda bruta da atividade leiteira (Gross income of dairy activity).

2 - Renda bruta do leite (Gross income of milk).

3 - Participação da renda bruta do leite na renda bruta da atividade leiteira (Proportion of gross income of milk in gross income of dairy activity)

4 - Preço do leite (Milk price).

5 - Gasto anual com concentrado para o rebanho (Annual expense with concentrate).

6 - Gasto com mão-de-obra na atividade leiteira (Expense with labor in the dairy activity).

7 - Participação do gasto com concentrado para o rebanho na renda bruta do leite (Proportion of concentrate expense in the gross income of milk).

8 - Participação do gasto com mão de obra na renda bruta do leite (Proportion of labor expense in the gross income of milk).

9 - Custo operacional efetivo da atividade leiteira (Effective operational cost of dairy activity).

10 - Custo operacional total da atividade leiteira (Total operational cost of dairy activity).

11 - Custo total da atividade leiteira (Total cost of dairy activity).

12 - Participação do custo operacional efetivo na renda bruta da atividade leiteira (Proportion of effective operational cost in the gross income of dairy activity).

13 - Participação do custo operacional total na renda bruta da atividade leiteira (Proportion of total operational cost in the gross income of dairy activity).

14 - Custo operacional efetivo do leite (Effective operational cost of milk).

15 - Custo operacional total do leite (Total operational cost of milk).

16 - Custo total do leite(Total cost of milk).

livre mobilidade de empresas (Reis, 2002), a tendência é a manutenção da situação de lucro normal. Nesta condição, a longo prazo, os produtores que estão na atividade permanecem em operação, enquanto os que estão ausentes não são atraídos.

Observou-se que, em média, os produtores estavam operando com lucro supernormal, o que indica tendência de expansão da atividade, seja pelo crescimento das atuais unidades seja pela entrada de novos produtores. Esse resultado está de acordo com a situação observada na região, que apresentou crescimento de $2,72 \%$ ao ano entre 1995 e 2004, superior à média estadual de 2,35\% ao ano, consolidando-se como a maior bacia leiteira do estado da Bahia (IBGE, 2005).

Na Tabela 6 observam-se as estatísticas descritivas dos indicadores de rentabilidade das empresas que exploram pecuária de leite no Extremo Sul da Bahia. A análise da taxa de remuneração do capital investido permite a mesma interpretação da análise de lucro, com a vantagem de que a taxa de retorno é determinada, a posteriori, enquanto, na análise de lucro, a taxa de remuneração do capital é arbitrada anteriormente para o cálculo de custo total.
Desse modo, permite comparar atratividades financeiras de diferentes negócios. Observou-se que, em média, a taxa de remuneração do capital investido foi superior à taxa real (taxa nominal descontada a inflação) de oportunidade adotada, de $4,0 \%$ ao ano.

$\mathrm{Na}$ Tabela 7 são apresentados os coeficientes de correlação e os níveis descritivos de probabilidade (Valor-P) dos indicadores avaliados com a taxa de remuneração do capitalinvestido (\% ao ano). Entre os fatores de produção, o recurso terra apresentou maiores correlações que os recursos mão-de-obra e animais, comprovando a importância da produtividade deste recurso no desempenho econômico da atividade leiteira.

A correlação $(\mathrm{P}<0,10)$ positiva entre produção de leite e taxa de remuneração do capital investido indica que, possivelmente, os produtores estão operando na fase de economia de escala (Ferguson, 1996). Nesta fase, o aumento da produção gera aumento menos que proporcional no custo total e, conseqüentemente, crescimento mais que proporcional no lucro da atividade. Este resultado reforça a importância do aumento no volume de produção de leite na atratividade do negócio (Schiffler et al., 1999; Gomes, 2005a). 
Tabela 5 - Estatísticas descritivas da margem bruta, da margem líquida e do lucro de empresas que exploram pecuária leiteira no Extremo Sul da Bahia

Table 5 - Descriptive statistics of the gross margin, net margin and profit of dairy farms in the Extremo Sul da Bahia

\begin{tabular}{|c|c|c|c|c|c|}
\hline $\begin{array}{l}\text { Especificação } \\
\text { Item }\end{array}$ & $\begin{array}{l}\text { Unidade } \\
\text { Unity }\end{array}$ & $\begin{array}{l}\text { Média } \\
\text { Mean }\end{array}$ & $\begin{array}{l}\text { Mínimo } \\
\text { Minimum }\end{array}$ & $\begin{array}{l}\text { Máximo } \\
\text { Maximum }\end{array}$ & $\begin{array}{l}\mathrm{DP} \\
S D\end{array}$ \\
\hline 1 & $\mathrm{R} \$$ /ano (R\$year) & $53.804,56$ & $24.363,00$ & $74.374,00$ & $15.466,45$ \\
\hline 2 & $\mathrm{R} \$$ /ano ( $R \$$ /year) & $42.428,00$ & $11.513,00$ & $57.328,00$ & $14.453,98$ \\
\hline 3 & $\mathrm{R} \$$ /ano ( $R \$$ /year) & $10.844,22$ & $-23.479,00$ & $26.527,00$ & $15.930,38$ \\
\hline 4 & $\mathrm{R} \$ / \mathrm{L}$ & 0,2085 & 0,1680 & 0,2656 & 0,0275 \\
\hline 5 & $\mathrm{R} \$ / \mathrm{L}$ & 0,1644 & 0,0794 & 0,2121 & 0,0355 \\
\hline 6 & $\mathrm{R} \$ / \mathrm{L}$ & 0,0420 & $-0,1619$ & 0,0977 & 0,0789 \\
\hline 7 & $\mathrm{R} \$ /$ vaca/ano ( $R$ \$/cows/year) & 349,13 & 232,03 & 489,45 & 87,47 \\
\hline 8 & $\mathrm{R} \$ /$ vaca/ano $(R \$ /$ cows/year $)$ & 275,31 & 109,65 & 413,46 & 219,23 \\
\hline 9 & $\mathrm{R} \$ / \mathrm{vaca} /$ ano $(R \$ /$ cows/year $)$ & 70,37 & $-223,61$ & 219,23 & 127,27 \\
\hline 10 & $\mathrm{R} \$ /$ ha/ano $(R \$ /$ ha/year $)$ & 253,27 & 93,70 & 413,19 & 99,54 \\
\hline 11 & $\mathrm{R} \$ /$ ha/ano ( $R \$ /$ ha/year) & 199,72 & 44,28 & 322,77 & 89,51 \\
\hline 12 & $\mathrm{R} \$ / \mathrm{ha} / \mathrm{ano}(R \$ /$ ha/year $)$ & 51,05 & $-90,30$ & 171,14 & 78,89 \\
\hline
\end{tabular}

1 - Margem bruta da atividade leiteira (Gross margin of dairy activity).

2 - Margem líquida da atividade leiteira (Net margin of dairy activity).

3 - Lucro da atividade leiteira (Profit of dairy activity).

4 - Margem bruta do leite (Gross margin of milk).

5 - Margem líquida do leite (Net margin of milk).

6 - Lucro do leite(Profit of milk).

7 - Margem bruta da atividade leiteira por total de vacas (Gross margin of dairy activity per total cows).

8 - Margem líquida da atividade leiteira por total de vacas (Net margin of dairy activity per total cows).

9 - Lucro da atividade leiteira por total de vacas (Profit of dairy activity per total cows).

10 - Margem bruta da atividade leiteira por área (Gross margin of dairy activity per area).

11 - Margem líquida da atividade leiteira por área (Net margin of dairy activity per area).

12 - Lucro da atividade leiteira por área (Profit of dairy activity per area).

Tabela 6 - Estatísticas descritivas dos indicadores de rentabilidade das empresas que exploram pecuária leiteira no Extremo Sul da Bahia Table 6 - Descriptive statistics of the indicators of profitability of dairy farms in the Extremo Sul da Bahia

\begin{tabular}{|c|c|c|c|c|c|}
\hline $\begin{array}{l}\text { Especificação } \\
\text { Item }\end{array}$ & $\begin{array}{l}\text { Unidade } \\
\text { Unity }\end{array}$ & $\begin{array}{l}\text { Média } \\
\text { Mean }\end{array}$ & Mínimo & Máximo & DP \\
\hline & & & & & \\
\hline 1 & $\%$ ao ano (\% per year) & 5,37 & 1,32 & 8,71 & 2,10 \\
\hline
\end{tabular}

1 - Taxa de remuneração do capital investido (Remuneration rate of capital invested).

2 - Capital investido na atividade em relação a produção diária de leite (Capital invested to daily production of milk ratio).

Apesar da larga adoção da relação de vacas em lactação por total de vacas como critério de monitoramento do sistema de produção, o estudo demonstrou que não houve correlação $(\mathrm{P}>0,10)$ deste indicador com o desempenho econômico da atividade leiteira. Todavia, a relação de vacas em lactação pelo total do rebanho apresentou correlação positiva com o desempenho econômico $(\mathrm{P}<0,10)$, evidenciando que a utilização deste índice global é mais eficaz que o anterior como critério de avaliação zootécnica.

Entre os indicadores clássicos utilizados como referência na avaliação de sistemas de produção de leite (Gomes, 2000) - gasto com concentrado para o rebanho em relação à renda bruta do leite, gasto com mão-de-obra em relação à renda bruta do leite, participação do custo operacional efetivo e do custo operacional total da atividade na renda bruta da atividade -, apenas o primeiro indicador não esteve correlacionado $(\mathrm{P}>0,10)$ à taxa de remuneração do capital investido.
O indicador capital investido em relação à produção diária de leite esteve alta e negativamente correlacionado $(\mathrm{P}<0,10)$ à rentabilidade do sistema, o que reforça as observações de Gomes (2005a) sobre a eficácia desse indicador como critério de avaliação econômica em sistemas de produção de leite.

Na Tabela 8 são descritos as estimativas de parâmetros de regressão, os níveis descritivos de probabilidade (Valor-P) e os coeficientes de determinação $\left(\mathrm{r}^{2}\right)$ dos indicadores-referência avaliados em relação à taxa de remuneração do capital investido (\% ao ano).

$\mathrm{Na}$ Tabela 9 são apresentados os valores de indicadores-referência das empresas que exploram a pecuária leiteira no Extremo Sul da Bahia em quatro cenários de taxa de remuneração do capital investido (4, 6, 8 e $10 \%$ ao ano). O volume mínimo de produção para viabilizar a atividade (456 a $703 \mathrm{~L} / \mathrm{dia}$ ) foi menor que o observado em sistemas de maior produtividade por vaca (Schiffler et al., 1999; Ferreira, 2002), 
Tabela 7 - Coeficientes de correlações e níveis descritivos de probabilidade (Valor-P) dos indicadores avaliados com a taxa de remuneração do capital investido (\% ao ano)

Table 7 - Coefficients of correlations and descriptive levels of probability $(P$-Value) of the studied indicators with remuneration rate of invested capital (\% in the year)

\begin{tabular}{lcc}
\hline $\begin{array}{l}\text { Indice } \\
\text { Index }\end{array}$ & $\begin{array}{c}\text { Coeficiente de correlação } \\
\text { Coefficient of correlation }\end{array}$ & $\begin{array}{l}\text { Valor- } P \\
P \text {-Value }\end{array}$ \\
\hline 1 & 0,6160 & 0,0387 \\
2 & 0,1614 & 0,3391 \\
3 & 0,2292 & 0,2765 \\
4 & $-0,1487$ & 0,3513 \\
5 & $-0,3409$ & 0,1847 \\
6 & 0,2630 & 0,2471 \\
7 & 0,9477 & 0,0001 \\
8 & 0,6407 & 0,0315 \\
9 & 0,3556 & 0,1738 \\
10 & 0,4658 & 0,1032 \\
11 & 0,6875 & 0,0204 \\
12 & 0,3462 & 0,1807 \\
13 & 0,5590 & 0,0588 \\
14 & 0,4848 & 0,0930 \\
15 & $-0,2720$ & 0,2394 \\
16 & $-0,5395$ & 0,0669 \\
17 & $-0,8344$ & 0,0026 \\
18 & 0,4359 & 0,1204 \\
19 & $-0,8134$ & 0,0038 \\
20 & $-0,9400$ & 0,0001 \\
\hline
\end{tabular}

1 - Produção diária de leite (Daily milk production).

2 - Número total de vacas (Total number of cows).

3 - Número de vacas em lactação (Number of lactating cows).

4 - Total de animais no rebanho (Total herd).

5 - Total de unidade animais (Total animal units).

6 - Mão-de-obra total (Total labor).

7 - Produtividade da terra (Land productivity)

8 - Número de vacas em lactação por área (Numberof lactating cows per area).

9 - Taxa de lotação ( Stocking rate).

10 - Produtividade por vaca em lactação (Productivity per lactating cow).

11 - Produtividade por total de vaca (Productivity per total cow).

12 - Relação de vacas em lactação por total de vacas(Lactating cows to total cows ratid.

13 - Relação de vacas em lactação por total do rebanho (Lactating cows to total herd ratio.

14 - Produtividade da mão-de-obra (Labor productivity).

15 - Produtividade do concentrado (Concentrate productivity).

16 - Participação do custo operacional efetivo na renda bruta da atividade leiteira (Proportion of effective operational cost in the gross income of dairy activity).

17 - Participação do custo operacional total na renda bruta da atividade leiteira (Proportion of total operationalcost in the gross income of dairy activity).

18 - Participação do gasto com concentrado para o rebanho na renda bruta do leite (Proportion of the concentrate expense per herd in the gross income of milk).

19 - Participação do gasto com mão-de-obra na renda bruta do leite (Proportion of labor expense per herd in the gross income of milk).

20 - Capital investido na atividade em relação à produção diária de leite (Capital invested to daily production of milk ratio). o que indica que a lucratividade do negócio depende da combinação de produtividade e volume de produção (Gomes, 2000). Aumentos da produtividade do rebanho não acompanhados de elevação expressiva no volume produzido podem não trazer os benefícios econômicos esperados.

Os valores dos indicadores-referência obtidos neste estudo diferem daqueles encontrados por Gomes (2000, 2005a, b, c) para produtores de leite de Minas Gerais. Nesse estado, os produtores bem sucedidos desembolsam até 30 e $20 \%$ da renda bruta do leite com alimentação concentrada para o rebanho e mão-de-obra permanente, respectivamente. O mesmo comportamento pode ser observado para os indicadores produtividade do fator terra, produtividade da mão-de-obra e capital investido na atividade em relação à produção diária de leite.

Para alcançar taxas de remuneração de $8 \%$ ao ano, a produtividade da mão-de-obra e da terra devem ser de $137 \mathrm{~L} /$ dia-homem e $1.284 \mathrm{~L} / \mathrm{ha} / \mathrm{ano}$, respectivamente. Os valores foram inferiores aos encontrados em uma amostra de 250 produtores no estado de Minas Gerais em 2004/2005, onde, para alcançar a mesma rentabilidade, precisaram de produtividades da mão-de-obra e terra superiores a $300 \mathrm{~L} /$ dia-homem e $3.500 \mathrm{~L} / \mathrm{ha} /$ ano (Gomes, 2005c).

A relação capital investido sobre a produção diária de leite, para atingir rentabilidade de $6 \%$ ao ano, deve ser de $\mathrm{R} \$ 1.508 / \mathrm{L}-\mathrm{dia}$, superior ao exigido pelos produtores do estado de Minas Gerais de R \$ 538/L-dia, (Gomes, 2005a). Isso significa que os sistemas analisados neste trabalho podem ser menos intensivos na utilização dos recursos produtivos, sem perder a competitividade.

Considerando um capital imobilizado de $\mathrm{R} \$ 500.000,00$ na atividade leiteira, a produção de leite necessária para atingir taxas de remuneração de capital investido, de 4, 6, 8 e $10 \%$ ao ano, deve ser 239 ( $\mathrm{R} \$ 500.000 / 2.093 \mathrm{R} \$ / \mathrm{L}$-dia), 332 ( $\mathrm{R} \$ 500.000 / 1.508 \mathrm{R} \$ / \mathrm{L}$-dia), 541 ( $\mathrm{R} \$ 500.000 / 924 \mathrm{R} \$ / \mathrm{L}$-dia) e 1.475 (R $\$ 500.000 / 339 \mathrm{R} \$ / \mathrm{L}$-dia) L/dia, respectivamente.

As razões das discrepâncias entre os valores de indicadores-referência observadas neste trabalho e os 
Tabela 8 - Parâmetros de regressão, níveis descritivos de probabilidade (Valor-P) e coeficientes de determinação $\left(r^{2}\right)$ dos indicadoresreferência econômicos, zootécnicos e de tamanho em relação à taxa de remuneração do capital investido (TRC, em \% ao ano)

Table 8 - Estimates of regression parameters, descriptive levels of probability ( $P$-value) and determination coefficients $(r 2)$ of indicators of size, productivity and economy in relation to remuneration rate of invested capital (\% per year)

\begin{tabular}{|c|c|c|c|c|}
\hline $\begin{array}{l}\text { Variável dependente } \\
\text { Dependent variable }\end{array}$ & $\begin{array}{l}\text { Unidade } \\
\text { Unit }\end{array}$ & $\begin{array}{l}\text { Parâmetro de regressão } \\
\text { Regression parameter }\end{array}$ & $\begin{array}{l}\text { Valor-P } \\
P \text {-value }\end{array}$ & $r^{2}$ \\
\hline 1 & $\mathrm{~L} /$ dia (L/year) & $\mathrm{Y}=291,17+41,20 \mathrm{TRC}$ & 0,0387 & 0,3794 \\
\hline 2 & L/ha/ano (L/ha/year) & $\mathrm{Y}=181,71+137,77 \mathrm{TRC}$ & 0,0001 & 0,8980 \\
\hline 3 & Vacas/ha (Cows/ha) & $\mathrm{Y}=0,20+0,04 \mathrm{TRC}$ & 0,0315 & 0,4105 \\
\hline 4 & $\mathrm{~L} / \mathrm{vaca} / \mathrm{dia}(L /$ cow/year $)$ & $\mathrm{Y}=1,99+0,25 \mathrm{TRC}$ & 0,0204 & 0,4726 \\
\hline 5 & $\%$ & $\mathrm{Y}=18,83+1,38 \mathrm{TRC}$ & 0,0588 & 0,3124 \\
\hline 6 & L/d.h. & $\mathrm{Y}=84,98+6,45 \mathrm{TRC}$ & 0,0930 & 0,2350 \\
\hline 7 & $\%$ & $\mathrm{Y}=65,46-1,48 \mathrm{TRC}$ & 0,0669 & 0,2911 \\
\hline 8 & $\%$ & $\mathrm{Y}=83,59-2,99 \mathrm{TRC}$ & 0,0026 & 0,6962 \\
\hline 9 & $\%$ & $\mathrm{Y}=40,66-2,31 \mathrm{TRC}$ & 0,0038 & 0,6616 \\
\hline 10 & $\mathrm{R} \$ / \mathrm{L}-\mathrm{dia}$ & $\mathrm{Y}=3.261,99-292,26 \mathrm{TRC}$ & 0,0001 & 0,8837 \\
\hline
\end{tabular}

1 - Produção diária de leite(Daily milk production).

2 - Produtividade da terra (Land productivity).

3 - Número de vacas em lactação por área (Number of lactating cows per area).

4 - Produtividade por total de vaca (Productivity per total cow).

5 - Relação de vacas em lactação por total do rebanho (Ratio of lactating cows to total herd.

6 - Produtividade da mão-de-obra (Labor productivity).

7 - Participação do custo operacional efetivo na renda bruta da atividade leiteira (Proportion of effective operational cost in the gross income of dairy activity

8 - Participação do custo operacional total na renda bruta da atividade leiteira (Proportion of total operational gross cost in the income of dairy activity).

9 - Participação do gasto com mão de obra na renda bruta do leite (Proportion of labor expense per herd in the gross income of milk).

10 - Capital investido na atividade em relação a produção diária de leite (Capital invested to daily production of milk ratio).

Tabela 9 - Indicadores-referência das empresas que exploram pecuária leiteira no Extremo Sul da Bahia em quatro cenários de taxa de remuneração do capital investido (4, 6, 8 e 10\% ao ano)

Table 9 - Values of indicator-reference of farms that explore livestock for milk Extremo Sul da Bahia, inrelating to four sceneries of invested rate of capital remuneration $(4,6,8$ and $10 \%$ in the year)

\begin{tabular}{|c|c|c|c|c|c|}
\hline \multirow[t]{2}{*}{$\begin{array}{l}\text { Indicador-referência } \\
\text { Reference-indicator }\end{array}$} & \multirow[t]{2}{*}{$\begin{array}{l}\text { Unidade } \\
\text { Unity }\end{array}$} & \multicolumn{4}{|c|}{$\begin{array}{l}\operatorname{TRC}^{1}(\% \text { ao ano }) \\
R T C^{1}(\% \text { per year })\end{array}$} \\
\hline & & 4 & 6 & 8 & 10 \\
\hline 1 & L/dia (L/year) & 456 & 538 & 621 & 703 \\
\hline 2 & L/ha/ano(L/ha/year) & 733 & 1.008 & 1.284 & 1.559 \\
\hline 3 & Vacas/ha (Cows/ha) & 0,37 & 0,45 & 0,54 & 0,62 \\
\hline 4 & $\mathrm{~L} /$ vaca/dia (L/cow/year) & 3,01 & 3,52 & 4,03 & 4,54 \\
\hline 5 & $\%$ & 24 & 27 & 30 & 33 \\
\hline 6 & L/d.h. & 111 & 124 & 137 & 150 \\
\hline 7 & $\%$ & 60 & 57 & 54 & 51 \\
\hline 8 & $\%$ & 72 & 66 & 60 & 54 \\
\hline 9 & $\%$ & 31 & 27 & 22 & 18 \\
\hline 10 & $\mathrm{R} \$ / \mathrm{L}-\mathrm{dia}$ & 2.093 & 1.508 & 924 & 339 \\
\hline
\end{tabular}

1 - Produção diária de leite(Daily milk production).

2 - $\quad$ Produtividade da terra (Land productivity).

3 - Número de vacas em lactação por área (Number lactating cows per area).

4 - Produtividade por total de vaca(Productivity per total cow).

5 - Relação de vacas em lactação por total do rebanho (Ratio of lactating cows to total herd.

6 - Produtividade da mão-de-obra (Labor productivity).

7 - Participação do custo operacional efetivo na renda bruta da atividade leiteira (Proportion of effective operational cost in the gross income of dairy activity).

8 - Participação do custo operacional total na renda bruta da atividade leiteira (Proportion of total operational cost in the gross income of dairy activity).

9 - Participação do gasto com mão de obra na renda bruta do leite (Proportion of labor expense per herd in the gross income of milk).

10 - Capital investido na atividade em relação a produção diária de leite (Capital invested to daily production of milk ratio).

${ }^{1}$ TRC - Taxa de remuneração do capital investido (Remuneration rate of invested capital).

descritos na literatura provavelmente se devem a diferenças socioeconômicas, culturais e edafo-climáticas inerentes aos ambientes em que os sistemas estão inseridos, o que remete a necessidade de avaliações regionalizadas.

\section{Conclusões}

A pecuária leiteira é uma atividade rentável mesmo em sistemas menos intensivos na utilização dos recursos 
produtivos terra, mão-de-obra e animais, porém exige elevada disponibilidade de terra, o que pode limitar sua adoção em larga escala.

Indicadores-referência obtidos para o Extremo Sul da Bahia diferem dos índices globais divulgados pelo setor, o que indica a necessidade de estudos regionalizados. Além disso, considerando o caráter dinâmico do ambiente que envolve os sistemas de produção de leite, é necessária a identificação e quantificação periódica desses índices.

A identificação de sistemas reais de produção de leite e a caracterização dos indicadores de maior correlação com a eficiência econômica poderão trazer maior embasamento para o debate sobre a viabilidade econômica na pecuária leiteira.

Além da quantificação de indicadores técnicos e econômicos, a metodologia utilizada pode ser aplicada para identificar os melhores processos de produção, bem como o perfil gerencial dos empresários, permitindo a utilização mais eficaz desta ferramenta.

\section{Literatura Citada}

ALVAREZ, G.A.; NOGUEIRA NETO, V.; MARTINS, P.C. et al. Perspectivas para o cooperativismo de leite no Brasil. In: VILELA, D.; BRESSAN, M.; FERNANDES, E.N. et al. (Eds.). Gestão ambiental e políticas para o agronegócio do leite. Juiz de Fora: Embrapa Gado de Leite, 2003. p.59-80.

BARGO, F.; MULLER, L.D.; KOLVER, E.S. et al. Invited review: production and digestion of supplemented dairy cows on pasture. Journal of Dairy Science, v.86, n.1, p.1-42, 2003.

BRESSAN, M.; VERNEQUE, R.S.; MOREIRA, P. A pecuária de leite em Goiás. Juiz de Fora: Embrapa Gado de Leite, Goiânia: Sindileite/Faeg, 1999. 310p.

DAVISON, T.M.; ELLIOTT, R. Response of lactating cows to grainbased concentrates in northern Australia. Tropical Grasslands, v.27, p.229-237, 1993.

DERESZ, F. Produção de leite de vacas mestiças holandês x zebu em pastagens de capim elefante, manejadas em sistema rotativo com e sem suplementação durante a época das chuvas. Revista Brasileira de Zootecnia, v.30, n.1, p.194-204, 2001.

DIAGNÓSTICO DA PECUÁRIA LEITEIRA DO ESTADO DE MINAS GERAIS. Relatório de pesquisa: tabelas. Belo Horizonte: SEBRAE-MG; FAEMG, 1996. 212p.

DÜRR, J.W. Como produzir leite de alta qualidade. Brasília: SENAR, 2005. 28p.

FERGUSON, C.E. Microeconomia. 19.ed. Rio de Janeiro: Florence Universitária, 1996. 610p.

FERREIRA, A.H. Eficiência de sistemas de produção de leite: uma aplicação da análise envoltória de dados na tomada de decisão. Viçosa, MG: Universidade Federal de Viçosa, 2002. 122p. Dissertação (Mestrado em Economia Aplicada) Universidade Federal de Viçosa, 2002.

FGVDADOS. [2005]. Informações econômicas on-line. Disponível em: <http://www.fgvdados.com.br/> Acesso em: $01 / 12 / 2005$
GOMES, S.T. Economia da produção de leite. Belo Horizonte: Itambé, 2000. 132p.

GOMES, S.T. Capital investido na produção de leite. Jornal da Produção de Leite. Convênio Nestlé/Funarbe/UFV. Ano XV, n. 191, janeiro/2005a

GOMES, S.T. [2005b]. Benchmark da produção de leite em MG. Disponível em: http://www.milkpoint.com.br/mn/ espacoabertoartigo.asp?nv=1\&id_artigo $=23393 \&$ area $=23 \&$ perM= $12 \&$ per $\mathrm{A}=2005$. Acesso em: 01/12/2005

GOMES, S.T. Intensificar ou não o sistema de produção de leite. In: INTERLEITE - ESTRATÉGIA E COMPETITIVIDADE NA CADEIA DE PRODUÇÃO DE LEITE, 7., 2005, Uberlândia. Anais... Uberlândia: Editora Berthier, 2005c. p.13-18.

GOMIDE, J.A. Produção de leite em regime de pasto. Revista Brasileira de Zootecnia, v.22, n.4, p.591-613, 1993.

HÍDRICOS [2003]. Disponível em: <www.hidricos.mg.gov.br/ extresul. Acesso em: 23/03/2003.

HOFFMANN, R.; SERRANO, O.; NEVES, E.M. et al. Administração da empresa agrícola. 5.ed. São Paulo: Pioneira, 1987. 325p.

INSTITUTO BRASILEIRO DE GEOGRAFIA E ESTATÍSTICA IBGE. [1996]. Censo agropecuário de 1995-1996. Disponível em: <http://www.ibge.gov.br/> Acesso em: 01/11/2005.

INSTITUTO BRASILEIRO DE GEOGRAFIA E ESTATÍSTICA IBGE. [2005]. Banco de dados. Disponível em: <http:// www.sidra.ibge.gov.br/bda/tabela/listabl.asp?c $=74 \& z=t \& o=20$ Acesso em: 10/12/2005.

KRUG, E.E.B. Estudo para identificação de benchmarking em sistemas de produção de leite no Rio Grande do Sul Porto Alegre: Universidade Federal do Rio Grande do Sul, 2001. 191p. Dissertação (Mestrado em Administração para Executivos) - Universidade Federal do Rio Grande do Sul, 2001.

LANA, R.P.; GOES, R.H.T.B.; MOREIRA, L.M. et al. Nova sistemática de avaliação de respostas produtivas (crescimento animal, vegetal, microbiano e produção de leite) ao suprimento variável de nutrientes. In: LANA, R.P. (Ed.). Nutrição e alimentação animal (mitos e realidade). Viçosa, MG: Editora UFV, 2005. p.265-292.

MADALENA, F.R.; TEODORO, R.L.; LEMOS, A.M. et al. Evaluation of strategies for crossbreeding of dairy cattle in Brazil. Journal of Dairy Science, v.73, n.7, p.1887-1901, 1990.

OLIVEIRA, H.T.V.; REIS, R.B.; JOANA, R.G. Comportamento da lactação de vacas mestiças $F 1$ holandês x zebu. Informe Agropecuário, v.25, n.221, p.73-79, 2004.

REIS, R.P. Fundamentos de economia aplicada. Lavras: UFLA/ FAEPE, 2002. 95p.

RIBAS, N.P.; PAULA, M.C.; ANDRADE, U.V.C. Contagem e escore de células somáticas em amostras de leite de tanques nos Estados de Santa Catarina, Paraná e São Paulo. In: BRITO, J.R.F.; PROTUGAL, J.A.B (Eds.). Diagnóstico da qualidade do leite, impacto para a indústria e a questão dos resíduos de antibióticos. Juiz de Fora: Embrapa Gado de Leite; Epamig/ CT/ILCT, 2003. p. 27-38.

SCHIFFLER, E.A.; MÂNCIO, A.B.; GOMES, S.T. et al. Efeito da escala de produção nos resultados da produção de leite $B$ no estado de São Paulo, Revista Brasileira de Zootecnia, v.28, n.2, p.425-431, 1999.

UNIVERSIDADE FEDERAL DE VIÇOSA - UFV.SAEG - Sistema de análises estatísticas e genéticas. Versão 7.1. Viçosa, MG. (Manual do usuário), 1997. 150p.

Recebido: 19/12/05 Aprovado: 16/10/06 